\title{
Concurrent arterial aneurysms in brain arteriovenous malformations with haemorrhagic presentation
}

\author{
C Stapf, J P Mohr, J Pile-Spellman, R R Sciacca, A Hartmann, H C Schumacher, H Mast
}

J Neurol Neurosurg Psychiatry 2002;73:294-298

See end of article for authors' affiliations

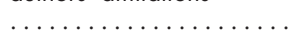

Correspondence to:

Dr C Stapf, Stroke Center,

The Neurological Institute,

710 West 168th Street,

New York, New York

10032, USA

christian.stapf@

medizin.fu-berlin.de

Received

18 February 2002

Accepted 23 April 2002

\begin{abstract}
Objective: To assess the effect of concurrent arterial aneurysms on the risk of incident haemorrhage from brain arteriovenous malformations (AVMs).

Methods: In a cross sectional study, 463 consecutive, prospectively enrolled patients from the Columbia AVM Databank were analysed. Concurrent arterial aneurysms on brain angiography were classified as feeding artery aneurysms, intranidal aneurysms, and aneurysms unrelated to blood flow to the AVM. Clinical presentation (diagnostic event) was categorised as intracranial haemorrhage proved by imaging or non-haemorrhagic presentation. Univariate and multivariate statistical models were applied to test the effect of age, sex, AVM size, venous drainage pattern, and the three types of aneurysms on the risk of AVM haemorrhage at initial presentation.

Results: Arterial aneurysms were found in 117 (25\%) patients with AVM (54 had feeding artery aneurysms, 21 had intranidal aneurysms, 18 had unrelated aneurysms, and 24 had more than one aneurysm type). Intracranial haemorrhage was the presenting symptom in 204 (44\%) patients with AVM. In the univariate model, the relative risk for haemorrhagic AVM presentation was $2.28195 \%$ confidence interval (CI) 1.12 to 4.64$)$ for patients with intranidal aneurysms and $1.88(95 \% \mathrm{Cl} 1.14$ to 3.08$)$ for those with feeding artery aneurysms. In the multivariate model an independent effect of feeding artery aneurysms (odds ratio $2.11,95 \% \mathrm{Cl} 1.18$ to 3.78) on haemorrhagic AVM presentation was found. No significant effect was seen for intranidal and unrelated aneurysms. The attributable risk of feeding artery aneurysms for incident haemorrhage in patients with AVM was $6 \%(95 \% \mathrm{Cl} 1 \%$ to $11 \%)$.

Conclusions: The findings suggest that feeding artery aneurysms are an independent determinant for increased risk of incident AVM haemorrhage.
\end{abstract}

ntracranial haemorrhage is the main cause of morbidity and mortality in patients with brain arteriovenous malformation (AVM). ${ }^{1}$ Several morphological, haemodynamic, and clinical factors associated with AVM haemorrhage have been identified. Among morphological determinants, small AVM size and deep venous drainage are best established..$^{2-4}$ The effect of concurrent arterial aneurysms-located in the AVM nidus, on feeding arteries, or on vessels unrelated to the arterial supply of the malformation-is as yet unresolved and the subject of ongoing discussions..$^{5-7}$ Their presence, however, influences treatment decisions (surgical, endovascular, or radiation treatment) and acute patient management (invasive versus conservative care).

Our aim was assessing the effect of different types of concurrent arterial aneurysms on haemorrhagic AVM presentation.

\section{SUBJECTS AND METHODS}

\section{Study subjects and data collection}

The Columbia AVM Databank is an ongoing prospective database collecting demographic, clinical, morphological, and treatment data on consecutive patients with brain AVM admitted to the Columbia-Presbyterian Medical Center since 1989. All malformations have been diagnosed based on brain imaging and cerebral angiography. Other types of intracranial fistulas (such as dural arteriovenous fistulas and vein of Galen malformations) are not included in the databank. Patients enrolled in the database are drawn from self and physician referrals from the New York metropolitan area, as well as from distant referral sites. Further details on the Columbia AVM Databank design, variable definitions, and methods have been described in prior publications ${ }^{89}$ and conform to the recently published consensus recommendations for AVM research reporting terminology. ${ }^{10}$
The clinical presentation (diagnostic event) was categorised as haemorrhagic or non-haemorrhagic. Haemorrhagic presentation (incident intracranial haemorrhage) was defined as a clinically symptomatic event with signs of fresh intracranial blood on head computed tomography or magnetic resonance imaging (MRI) or in the cerebrospinal fluid. Haemorrhage was inferred to be related to AVM whenever brain imaging showed topographic contiguity of the haematoma with the malformation or its feeding arteries. On imaging, the primary bleeding location was classified as being either intracerebral (with or without extension into the intraventricular or subarachnoid space) or extracerebral (intraventricular or subarachnoid without haemorrhage into brain parenchyma). Non-haemorrhagic AVM presentation was defined as any event (seizure, focal neurological deficit, headache, or other) unrelated to the AVM haemorrhage that led to the diagnosis of the AVM.

Morphological AVM features were evaluated by experienced neuroradiologists blinded to the patient's diagnostic event. Variables used in the present analysis were AVM size (measured as maximum nidus diameter in millimetres on pretreatment angiography or brain MRI), venous drainage pattern (categorised as angiographic drainage into the superficial cortical veins, drainage into the deep venous system, and combined superficial and deep drainage), and presence of concurrent arterial aneurysms. Arterial aneurysms were defined as saccular dilatations of the lumen $\geqslant 2$ times the width of the arterial vessel that carried the dilatation. They were further classified as feeding artery aneurysms, intranidal

Abbreviations: $\mathrm{AVM}$, arteriovenous malformation; $\mathrm{Cl}$, confidence interval; MRI, magnetic resonance imaging 
Table 1 Baseline characteristics of 463 patients with brain arteriovenous malformation (AVM)

\begin{tabular}{lr}
\hline Demographic characteristics & \\
Mean (SD) age at presentation (years) & $34(15)$ \\
Female sex & $255(55 \%)$ \\
Clinical presentation & $204(44 \%)$ \\
Intracranial haemorrhage & $133(29 \%)$ \\
Seizure* & $53(11 \%)$ \\
Headache* & $35(8 \%)$ \\
Neurological deficit* & $38(8 \%)$ \\
Other/asymptomatic* & $34(17)$ \\
Morphological characteristics & \\
Mean (SD) maximal AVM diameter (mm) & $226(49 \%)$ \\
Venous drainage pattern & $98(21 \%)$ \\
Superficial & $139(30 \%)$ \\
Deep & $117(25 \%)$ \\
Deep and superficial & \\
Concurrent arterial aneurysms & \\
\hline *Unrelated to haemorrhage. &
\end{tabular}

aneurysms, and aneurysms unrelated to blood flow to the AVM. A feeding artery was defined as any intracranial vessel that angiographically contributed arterial flow to the malformation. The AVM nidus was defined as the vascular mass included in the AVM size measurement. Intranidal aneurysms were coded when visualised early after angiographic injection, such as before substantial venous filling had occurred. Infundibula, arterial ectasias (dilated feeding vessels), and intranidal aneurysmal dilatations seen only during the venous angiographic phase were not coded as arterial aneurysms. Arterial aneurysms were coded as unrelated to the AVM when located on intracranial arteries not contributing blood flow to the AVM.

\section{Statistical analysis}

Standard univariate tests ( $\chi^{2}$ test, $t$ test) and multivariate logistic regression models controlling for age, sex, AVM size, deep venous drainage pattern, and the three types of aneurysms were applied to assess the independent effect of arterial aneurysms on haemorrhagic AVM presentation. The attributable risk of aneurysms to haemorrhagic presentation was determined as described by Fleiss ${ }^{11}$; the attributable risk (or aetiological fraction) measures the decrease in the overall proportion of haemorrhages expected from the elimination of aneurysms.

\section{RESULTS}

Table l summarises the demographic, clinical, and morphological characteristics of the study sample. Intracranial haemorrhage was the presenting symptom in 204 (44\%) patients with AVM; 132 of them presented with intracerebral, 34 with
Table 3 Multivariate logistic regression model testing the effect of aneurysm type on incident haemorrhage in 463 patients with AVM

\begin{tabular}{lllc}
\hline & Odds ratio & $95 \% \mathrm{Cl}$ & $\mathrm{p}$ Value \\
\hline Feeding artery aneurysm & 2.11 & 1.18 to 3.78 & 0.012 \\
Intranidal aneurysm & 1.83 & 0.79 to 4.21 & 0.158 \\
Unrelated aneurysms & 1.69 & 0.72 to 3.95 & 0.228 \\
Patient age & 1.00 & 0.99 to 1.01 & 0.923 \\
Female sex & 0.65 & 0.43 to 0.98 & 0.042 \\
AVM size* & 0.94 & 0.93 to 0.96 & $<0.0001$ \\
$\begin{array}{l}\text { Deep venous drainage } \\
\text { component }\end{array}$ & 2.42 & 1.58 to 3.69 & $<0.0001$ \\
\hline \multirow{3}{*}{ *Maximal diameter in millimetre increments. } & & \\
\hline
\end{tabular}

intraventricular, and 29 with subarachnoid haemorrhage. Because of missing data, the haemorrhage type was undefined in nine patients.

Of the $117(25 \%)$ patients with concurrent arterial aneurysms, 93 had a single aneurysm type ( 54 had feeding artery aneurysms, 21 had intranidal aneurysms, and 18 had aneurysms unrelated to flow to the AVM). The remaining 24 patients had more than one aneurysm type ( 10 with feeding artery and intranidal aneurysms, 10 with feeding artery and unrelated aneurysms, 1 with intranidal and unrelated aneurysms, and 3 with all three aneurysm types).

Concurrent arterial aneurysms were significantly more frequent in patients presenting with AVM haemorrhage than in those with a non-haemorrhagic AVM presentation (table 2). The difference remained significant $(\mathrm{p}<0.0001)$ in a multivariate model controlling for age, sex, AVM size, and deep venous drainage (odds ratio $3.17,95 \%$ confidence interval 1.91 to 5.28$)$.

\section{Feeding artery aneurysms}

Feeding artery aneurysms were detected in 77 (17\%) patients with AVM and were found significantly more often among patients presenting with intracranial haemorrhage than among those with non-haemorrhagic presentation (table 2). The difference remained significant in the multivariate model controlling for age, sex, AVM size, venous drainage pattern, and other concurrent aneurysm types (table 3 ). On the basis of these findings, the attributable risk of feeding artery aneurysms for incident haemorrhage in patients with AVM was estimated to be $6 \%$ (95\% confidence interval 1\% to $11 \%$ ) (fig 1).

Feeding artery aneurysms were significantly more frequent among the 29 patients presenting with subarachnoid haemorrhage $(52 \%, \mathrm{n}=15)$ than among the 132 patients with intracerebral haemorrhage $(17 \%, \mathrm{n}=23)$ or the 34 patients with intraventricular haemorrhage $(15 \%, \mathrm{n}=5, \mathrm{p}<0.001)$.

Table 2 Univariate tests of the effect of demographic and morphological characteristics and concurrent arterial aneurysms on incident haemorrhage in 463 patients with AVM

\begin{tabular}{|c|c|c|c|c|}
\hline & $\begin{array}{l}\text { Haemorrhagic AVM } \\
\text { presentation }(n=204)\end{array}$ & $\begin{array}{l}\text { Non-haemorrhagic AVM } \\
\text { presentation }(n=259)\end{array}$ & Odds ratio $(95 \% \mathrm{Cl})$ & $\mathrm{p}$ Value \\
\hline Mean (SD) age at presentation (years) & $35(16)$ & $33(14)$ & 1.01 (1.00 to 1.03$)$ & 0.060 \\
\hline Female sex & $105(51 \%)$ & $150(58 \%)$ & $0.76(0.53$ to 1.11$)$ & 0.153 \\
\hline Mean (SD) maximal AVM diameter (mm) & $28(17)$ & $40(15)$ & 0.95 (0.94 to 0.96$)$ & $<0.0001$ \\
\hline Deep venous drainage & $68(33 \%)$ & $30(12 \%)$ & 3.81 (2.29 to 6.32) & $<0.0001$ \\
\hline Deep and superficial venous drainage & $52(25 \%)$ & $87(34 \%)$ & $1.00(0.65$ to 1.55$)$ & 0.988 \\
\hline Concurrent aneurysm & $70(34 \%)$ & $47(18 \%)$ & 2.35 (1.53 to 3.60$)$ & $<0.0001$ \\
\hline Feeding artery aneurysm* & $44(22 \%)$ & $33(13 \%)$ & 1.88 (1.14 to 3.08$)$ & 0.013 \\
\hline Intranidal aneurysm* & $22(11 \%)$ & $13(5 \%)$ & 2.28 (1.12 to 4.64$)$ & 0.023 \\
\hline Unrelated aneurysm* & $15(7 \%)$ & $17(7 \%)$ & $1.13(0.55$ to 2.31$)$ & 0.748 \\
\hline
\end{tabular}

*The total number of patients in the three groups of aneurysm subtypes exceeds the total number of 117 cases with concurrent arterial aneurysms because 24 patients had multiple aneurysm types. $\mathrm{Cl}$, confidence interval. 

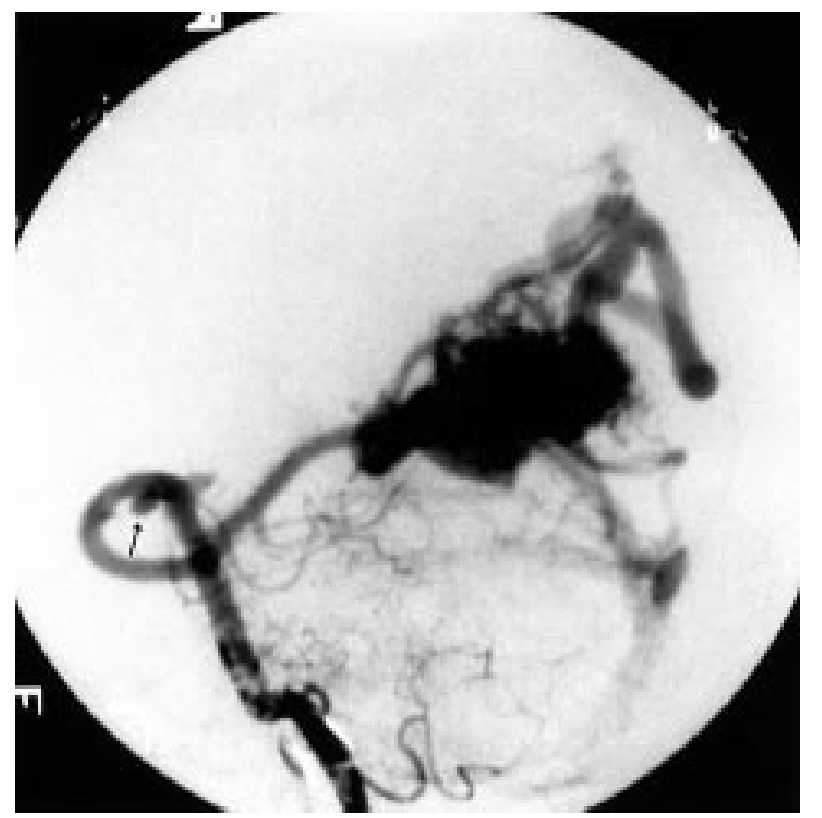

Figure 1 Feeding artery aneurysm (arrow) on a posterior cerebral artery feeding a right occipital brain arteriovenous malformation (vertebral artery injection)

\section{Intranidal aneurysms}

Intranidal aneurysms occurred in $35(8 \%)$ patients with AVM. By univariate comparison, intranidal aneurysms were detected significantly more often in patients who presented with intracranial haemorrhage than in those without a haemorrhagic presentation (table 2). The effect of intranidal aneurysms on AVM haemorrhage, however, was not significant in the multivariate model controlling for age, sex, AVM size, venous drainage pattern, and the two other aneurysm types (table 3) (fig 2).

No significant association was found for intranidal aneurysms with intracerebral (12\%), intraventricular $(15 \%)$, or subarachnoid haemorrhage $(3 \%, \mathrm{p}=0.3)$.
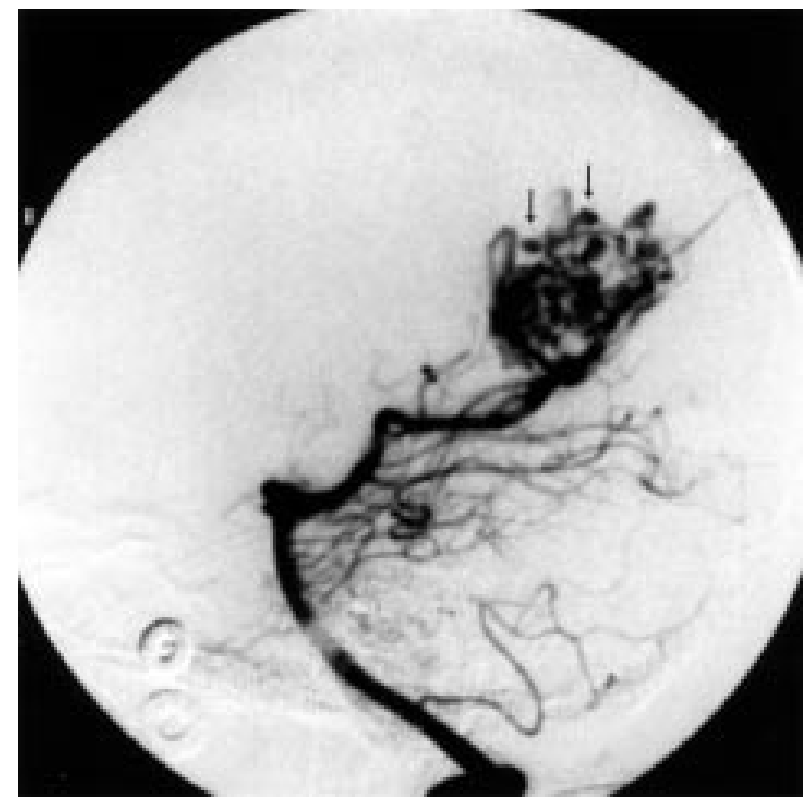

Figure 2 Intranidal aneurysms (arrows) in a right occipital arteriovenous malformation fed by posterior cerebral artery branches (early arterial phase, vertebral artery injection).

\section{Unrelated arterial aneurysms}

Thirty two (7\%) patients were found to have arterial aneurysms unrelated to blood flow to the AVM. No significant association between unrelated arterial aneurysms and AVM haemorrhage at initial presentation was found (table 2, table 3).

No significant association was found for unrelated aneurysms with intracerebral $(7 \%)$, intraventricular $(3 \%)$, or subarachnoid haemorrhage $(18 \%, \mathrm{p}=0.2)$.

Two patients presented with subarachnoid haemorrhage due to rupture of an aneurysm located on an artery not feeding the brain AVM. Their diagnostic angiography showed a brain AVM remote from the bleeding location. For the analyses presented above, these two patients were coded as having a non-haemorrhagic AVM presentation. Adding the two cases to the group of patients with haemorrhagic AVM presentation did not change the results for the effect of feeding artery, intranidal, or unrelated aneurysms on AVM haemorrhage.

\section{DISCUSSION}

Several retrospective studies suggested a higher risk of haemorrhage in patients with AVM with concurrent arterial aneurysms. ${ }^{212-16}$ The retrospective actuarial analysis by Brown et $\mathrm{al}^{17}$ showed an increased annual risk of $7.0 \%$ for intracranial haemorrhage in the setting of unruptured AVMs with a concurrent arterial aneurysm (any coexisting saccular aneurysm seen on brain angiography) compared with a $1.7 \%$ annual risk for patients harbouring an AVM without concurrent aneurysms. ${ }^{17}$ In a combined prospective and retrospective series of 632 patients with AVM, a significant association with haemorrhagic AVM presentation was found for intranidal but not for feeding artery aneurysms. ${ }^{6}$ In contrast, other retrospective ${ }^{4518}$ and prospective ${ }^{19} 20$ studies have found no independent effect of concurrent arterial aneurysms on the risk of intracranial haemorrhage.

Our own series provides clinical and morphological information drawn from one of the largest prospective AVM datasets available, thereby allowing univariate and multivariate risk analysis modelling. In the univariate analysis, our findings go beyond prior reports from retrospective patient samples and suggest that both intranidal and feeding artery aneurysms confer a significantly higher risk for incident intracranial haemorrhage (table 2). ${ }^{5-7}$ 12-19 The association of intranidal aneurysms with haemorrhagic AVM presentation, however, did not prevail in the multivariate model, where only feeding artery aneurysms were found to be an independent risk factor for haemorrhagic presentation. Given the smaller number of patients with intranidal aneurysms, a lack of statistical power may affect these findings; thus, our results do not negate the possibility of an effect of intranidal aneurysms on incident AVM haemorrhage.

In our analyses, the effect of established morphological risk factors for incident AVM haemorrhage such as AVM size and deep venous drainage remains stable and is comparable with data reported in prior AVM series. ${ }^{24-7}$ The findings that unrelated aneurysms do not affect the risk of AVM haemorrhage and that feeding artery aneurysms are associated with subarachnoid haemorrhage are biologically plausible and may lend credence to our main results.

The reported rates of concurrent aneurysms in patients with AVM vary from $3 \%{ }^{21}$ to $58 \%{ }^{22}$ Some of this variation may result from low interrater agreement. Recent data from an ongoing internet based validation study on AVM morphology suggested only poor agreement for the presence of AVM related aneurysms among independent international observers $(\kappa=0.29){ }^{23}$ Different study definitions of concurrent aneurysms may also contribute to variations in reported aneurysm rates. In a recent prospective AVM series in which there was a high detection rate $(46 \%)$ of concurrent 
aneurysms, infundibulae greater than $3 \mathrm{~mm}$ and arterial dilatations in the order of $1 \mathrm{~mm}$ were coded as arterial aneurysms. ${ }^{7}{ }^{19}$ Lastly, referral bias to tertiary treatment centres may influence the rate of detected aneurysms. ${ }^{24}$

The lower haemorrhage risk in women remains unexplained. A lower follow up haemorrhage risk ${ }^{8}$ and a higher risk for surgical treatment in women have been observed, ${ }^{25}$ suggesting sex differences in patients with AVM in both natural history and treatment risk.

The dichotomisation of presentation into haemorrhagic versus non-haemorrhagic in our study may raise concerns regarding the exact source of the bleeding (that is, rupture within the AVM nidus, or haemorrhage from a concurrent arterial aneurysm or a venous pouch). The non-invasive diagnostic evaluation (computed tomography, brain MRI, or cerebral angiography) of survivors of AVM haemorrhage in general (as well as of the patients in our study sample), however, precludes the determination of a definite bleeding source. Our data suggest an association between AVM haemorrhage and feeding artery aneurysms; they do not offer proof as to a definite bleeding source.

As to feeding artery aneurysms, current treatment recommendations for patients with AVM are non-uniform. Some authors propose that the arterial aneurysm be treated immediately, ${ }^{5}{ }^{13}$ while others consider the malformation to be the primary treatment target ${ }^{6}$ or suggest that both abnormalities be treated at the same time. ${ }^{27-29}$ Our findings suggest that feeding artery aneurysms constitute an independent risk factor for haemorrhagic AVM presentation and may therefore be considered for surgical or endovascular treatment or both. However, some limitations should caution against final conclusions regarding treatment recommendations.

Firstly, population based fatality rates after AVM haemorrhage are unknown and the overall frequency of AVM haemorrhage may be underestimated from referral centre patient cohorts, ${ }^{30}{ }^{31}$ leading to the possibility of a systematic error in the analyses. Secondly, the results of our study are based on incident intracranial haemorrhage. It has not been shown that feeding artery aneurysms are associated with subsequent haemorrhage, the primary target of prevention in patients with AVM. Thirdly, only $22 \%$ of our patients with incident intracranial haemorrhage had aneurysms on an AVM feeding vessel and only $52 \%$ of all patients presenting with subarachnoid haemorrhage had a feeding artery aneurysm. Given the prevalence of feeding artery aneurysms in our sample population, the attributable risk calculation suggests that only $6 \%$ of haemorrhages would have been prevented by eliminating all feeding artery aneurysms in the study sample. Lastly-in a strict sense-even for patients with AVM with feeding artery aneurysms and intracranial haemorrhage, the present findings leave open the question of whether the aneurysm is the biological cause of the haemorrhage or just a marker of general vascular changes secondary to flow abnormalities due to the AVM. The high rate of concurrent arterial aneurysms in patients with AVM has fostered hypotheses on a common underlying aetiology. One hypothesis, that there is a primary smooth muscle defect at the level of the affected arterial branches, ${ }^{12} 3233$ may be testable in patients with AVM with concurrent arterial aneurysms and deserves further study.

\section{ACKNOWLEDGEMENTS}

Supported in part by NIH grant R01 NS 40792-01 (PI: J P Mohr). The authors thank S Marshall, Dr B A Aagaard, and Dr W L Young for their help during data collection.

\section{Authors' affiliations \\ *C Stapf, J P Mohr, H C Schumacher, ${ }^{* *}$ H Mast, Stroke Center, The Neurological Institute, Columbia University College of Physicians and} Surgeons, New York, New York, USA
J Pile-Spellman, Interventional Neuroradiology, Columbia University College of Physicians and Surgeons

R R Sciacca, Department of Medicine, Columbia University College of Physicians and Surgeons

A Hartmann, Department of Neurology, Universitätsklinikum Benjamin Franklin, Freie Universität Berlin, Berlin, Germany

*Also the Department of Neurology, Universitätsklinikum Benjamin Franklin

**Also the Schlaganfallzentrum Halle, Stroke Unit, Neurologische Klinik, BG Kliniken Bergmannstrost, Halle/Saale, Germany

Competing interests: none declared

\section{REFERENCES}

1 The Arteriovenous Malformation Study Group. Arteriovenous malformations of the brain in adults. N Engl J Med 1999;340:1812-8.

2 Marks MP, Lane B, Steinberg GK, et al. Hemorrhage in intracerebral arteriovenous malformations: angiographic determinants. Radiology 1990;176:807-13

3 Duong HD, Young WL, Vang MC, et al. Feeding artery pressure and venous drainage pattern are primary determinants of hemorrhage from cerebral arteriovenous malformations. Stroke 1998;29:1167-76.

4 Langer DJ, Lasner TM, Hurst RW, et al. Hypertension, small size, and deep venous drainage are associated with risk of hemorrhagic presentation of cerebral arteriovenous malformations. Neurosurgery 1998;42:481-9

5 Thompson RC, Steinberg GK, Levy RP, et al. The management of patients with arteriovenous malformations and associated intracranial aneurysms. Neurosurgery 1998;43:202-12.

6 Redekop G, TerBrugge K, Montanera W, et al. Arterial aneurysms associated with cerebral arteriovenous malformation: classification, incidence, and risk of hemorrhage. J Neurosurg 1998;89:539-46.

7 Meisel HJ, Mansmann U, Alvarez H, et al. Cerebral arteriovenous malformations and associated aneurysms: analysis of 305 cases from a series of 662 patients. Neurosurgery 2000;46:793-802.

8 Mast $\mathbf{H}$, Young WL, Koennecke HC, et al. Risk of spontaneous hemorrhage after diagnosis of cerebral arteriovenous malformations. Lancet 1997:350:1065-8.

9 Hartmann A, Mast H, Mohr JP, et al. Morbidity of intracranial hemorrhage in patients with cerebral arteriovenous malformation. Stroke 1998;29:931-4.

10 Anon. Reporting terminology for brain arteriovenous malformation clinical and radiographic features for use in clinical trials. Joint Writing Group of the Technology Assessment Committee American Society of Interventional and Therapeutic Neuroradiology; Joint Section on Cerebrovascular Neurosurgery a Section of the American Association of Neurological Surgeons and Congress of Neurological Surgeons; Section of Stroke and the Section of Interventional Neurology of the American Academy of Neurology. Stroke 2001;32:1430-42.

11 Fleiss JL. Inference about population attributable risk from cross-sectional studies. Am J Epidemiol 1979;110:103-4.

12 Anderson RM, Blackwood W. The association of arteriovenous angioma and saccular aneurysm of the arteries of the brain. J Pathol Bacteriol 1959;77:101-9.

13 Batjer H, Suss RA, Samson D. Intracranial arteriovenous malformations associated with aneurysms. Neurosurgery 1986;18:29-35.

14 Lasjaunias $\mathbf{P}$, Piske R, TerBrugge K, et al. Cerebral arteriovenous malformations (C.AVM) and associated arterial aneurysms (AA): analysis of 101 C.AVM cases, with 37 AA in 23 patients. Acta Neurochir (Wien) 1988;91:29-36.

15 Turiman F, Massoud TF, Viñuela F, et al. Correlation of the angioarchitectural features of cerebral arteriovenous malformations with clinical presentation of hemorrhage. Neurosurgery 1995;37:856-62.

16 Westphal M, Grzyska U. Clinical significance of pedicle aneurysms on feeding vessels, especially those located in infratentorial arteriovenous malformations. J Neurosurg 2000;92:995-1001.

17 Brown RD, Wiebers DO, Forbes GS. Unruptured intracranial aneurysms and arteriovenous malformations: frequency of intracranial hemorrhage and relationship of lesions. J Neurosurg 1990;73:859-63.

18 Nataf F, Meder JF, Roux FX, et al. Angioarchitecture associated with hemorrhage in cerebral arteriovenous malformations: a prognostic statistical model. Neuroradiology 1997;39:52-8.

19 Mansmann U, Meisel J, Brock M, et al. Factors associated with intracranial hemorrhage in cases of cerebral arteriovenous malformations. Neurosurgery 2000;46:272-81.

20 Stefani MA, Porter PJ, terBrugge KG, et al. Angioarchitectural factors present in brain arteriovenous malformations associated with hemorrhagic presentation. Stroke 2002;33:920-4.

21 Paterson JH, McKissock W. A clinical survey of intracranial angiomas with special reference to their mode of progression and surgical treatment: a report of 110 cases. Brain 1956:79:233-66.

22 Turiman F, Massoud TF, Viñuela F, et al. Aneurysm related to cerebral arteriovenous malformations: superselective angiographic assessment in 58 patients. Am J Neuroradiol 1994;15:1601-5.

23 Stapf C, Hofmeister C, Mast $\mathrm{H}$, et al. The feasibilty of an internet web-based, international study on brain arteriovenous malformations (the AVM world study) [abstract]. Stroke 2000;31:322. 
24 Hofmeister C, Stapf C, Hartmann A, et al. Demographic, morphologic, and clinical characteristics of 1289 patients with brain arteriovenous malformation. Stroke 2000;31:1307-10.

25 Hartmann A, Stapf C, Hofmeister C, et al. Determinants of neurologic outcome after surgery for brain arteriovenous malformation. Stroke 2000;31:2361-4.

26 Piotin M, Ross IB, Weill A, et al. Intracranial arterial aneurysms associated with arteriovenous malformations: endovascular treatment. Radiology $2001 ; 220: 506-13$

27 Suzuki J, Onuma T. Intracranial aneurysms associated with arteriovenous malformations. J Neurosurg 1979;50:742-6.

28 Kondziolka D, Nixon BJ, Lasjaunias $P$, et al. Cerebral arteriovenous malformations with associated arterial aneurysms: hemodynamic and therapeutic considerations. Can J Neurol Sci 1988;15:130-4.
29 Deruty R, Mottolese C, Soustiel JF, et al. Association of cerebral arteriovenous malformation and cerebral aneurysm: diagnosis and management. Acta Neurochir 1990;107:133-9.

30 Brown RD Jr, Wiebers DO, Torner JC, et al. Incidence and prevalence of intracranial vascular malformations in Olmsted County, Minnesota, 1965 to 1992. Neurology 1996:46:949-52.

31 Stapf C, Labovitz DL, Sciacca RR, et al. Incidence of adult brain arteriovenous malformation hemorrhage in a prospective population-based stroke survey. Cerebrovasc Dis 2002;13:43-6. 32 Voigt K, Beck U, Reinshagen G. A complex cerebral vascular malformation studied by angiography: multiple aneurysms, angiomas and arterial ectasia. Neuroradiology 1973;5:117-23.

33 Miyasaka K, Wolpert SM, Prager RJ. The association of cerebral aneurysm, infundibula, and intracranial arteriovenous malformations. Stroke 1982;13:401-8.

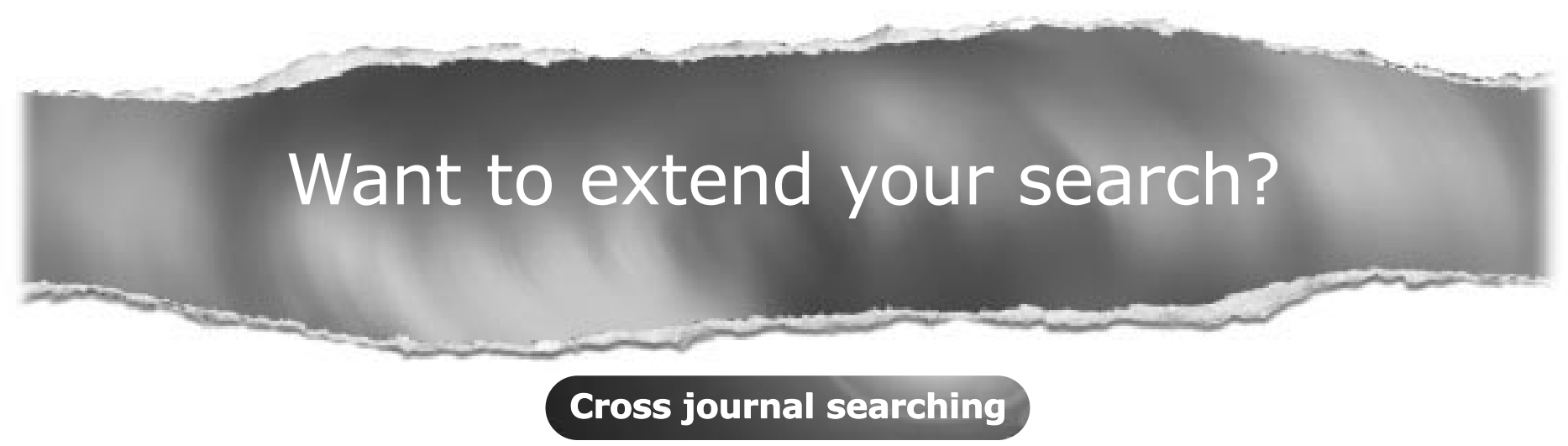

If you can't find what you are looking for in the Journal of Neurology, Neurosurgery, and Psychiatry you can extend your search across many of the more than 200 journals available for selection. You can restrict your search to specific subject areas (eg, clinical medicine, basic research), or select specific journals, or search all available titles.

www.jnnp.com 